\title{
Multi-spacecraft observations and transport modeling of energetic electrons for a series of solar particle events in August 2010
}

\author{
Wolfgang Dröge* \\ Institut für Theoretische Physik und Astrophysik, Universität Würzburg, 97074 Würzburg, \\ Germany \\ E-mail:droege@astro.uni-wuerzburg.de
}

Yulia Kartavykh

Institut für Theoretische Physik und Astrophysik, Universität Würzburg, 97074 Würzburg, Germany; Ioffe Physical-Technical Institute, St. Petersburg 194021, Russia

E-mail: ykartavykh@astro.uni-wuerzburg.de

\section{Andreas Klassen}

Christian-Albrechts-Universität, Kiel, Germany

E-mail:klassen@physik.uni-kiel.de

\section{Nina Dresing}

Christian-Albrechts-Universität, Kiel, Germany

E-mail:dresing@physik.uni-kiel.de

\section{David Lario}

The Johns Hopkins University, Applied Physics Laboratory, Laurel, MD 20723-6099, USA

E-mail: david.lario@jhuapl.edu

During August 2010 a series of solar particle events, originating from the adjacent active regions 11093 and 11099, was observed by the two STEREO as well as by near-Earth spacecraft. For the events occurring on the August 7 and 18 we combine in-situ and remote-sensing observations with predictions from our model of three-dimensional anisotropic particle propagation in order to investigate the physical processes which cause the large angular spreads of the energetic particles during these events. In particular, we address the effects of lateral transport of the electrons in the solar corona as well as due to diffusion perpendicular to the average magnetic field in the interplanetary medium. We also study the influence of two Coronal Mass Ejections and associated shock waves on the electron propagation, and a possible longitudinal variation in space of the transport conditions during the above period. For the August 18 event we additionally utilize electron observations from the MESSENGER spacecraft at a distance of $0.31 \mathrm{AU}$ from the Sun for an attempt to separate between radial and longitudinal dependencies in the transport process.

The 34th International Cosmic Ray Conference,

30 July- 6 August, 2015

The Hague, The Netherlands

\footnotetext{
* Speaker.
} 


\section{Introduction}

Solar energetic particle (SEP) events as can be observed in the Earth orbit are determined by a combination of the underlying acceleration, injection and transport conditions, respectively, and therefore carry fundamental information on these processes. The two STEREO spacecraft in combination with observations close to Earth provide a unique platform to investigate SEP events over a wide longitudinal range but at nearly the same heliocentric distance avoiding radial gradient effects. A series of SEP events in August 2010 originating from nearly the same source regions at the Sun lend themselves for a comprehensive investigation of the underlying injection and transport processes. We apply a propagation model allowing for changing transport conditions from event to event to shed some light on the main processes leading to the wide angular SEP distributions during these events. The fact that, as the active regions rotated over the visible disc of the Sun, the flares associated with the consecutive SEP events were variably well magnetically connected to STEREO-A/B and ACE/SDO/Earth, respectively, offers an interesting perspective to investigate the relative contributions of particle transport effects in the solar corona and in the interplanetary medium to the longitudinal variation of particle fluxes at $1 \mathrm{AU}$.

\section{Observations}

In this work we combine electron data from the SEPT instrument [1] of the IMPACT suite [2] on board STEREO and from the EPAM instrument on board ACE [3] The SEPT instruments measure electrons in the range of 45-400 keV. The LEFS60 telescope of the ACE/EPAM instrument provides electron measurements in the range of 40 to about $350 \mathrm{keV}$. For the analysis presented here we restrict ourselves to electrons from the three spacecraft in the energy range of approximately 65-105 keV. Both SEPT and EPAM provide directional intensity measurements which, in combination with magnetic field measurements by STEREO/MAG [4] and ACE/MAG [5] were used to determine pitch angle distributions of the electrons. Solar wind plasma data provided by the STEREO/PLASTIC [6] and ACE/SWEPAM [7] instruments were used to reconstruct the magnetic connections between the spacecraft and the solar corona. Figure 1 shows observations of energetic electrons and the solar wind speed obtained on STEREO-B, ACE, and STEREO-A during the period of 2010 July 31 through 2010 August 20, during which a series of interplanetary particle events was produced by solar active regions (AR) 11093 and 11099. In detail, the panels of the figure display as a function of time (from top to bottom): the omni-directional electron intensities and pitch-angle distributions (binned in four sectors) of the 3 spacecraft, followed by the solar wind speeds. Because of the limited space available we focus here on the electron events of August 7 and 18 during which SEPs were observed on all three spacecraft, and which were associated with flare/CME activity for which comprehensive measurements, including EIT-waves, type III and coronal and IP type II radio bursts are available.

\section{Interplanetary transport}

In this work we consider the interplanetary transport of energetic particles which are accelerated impulsively in the solar flares, undergo some kind of lateral transport in the solar corona to a 


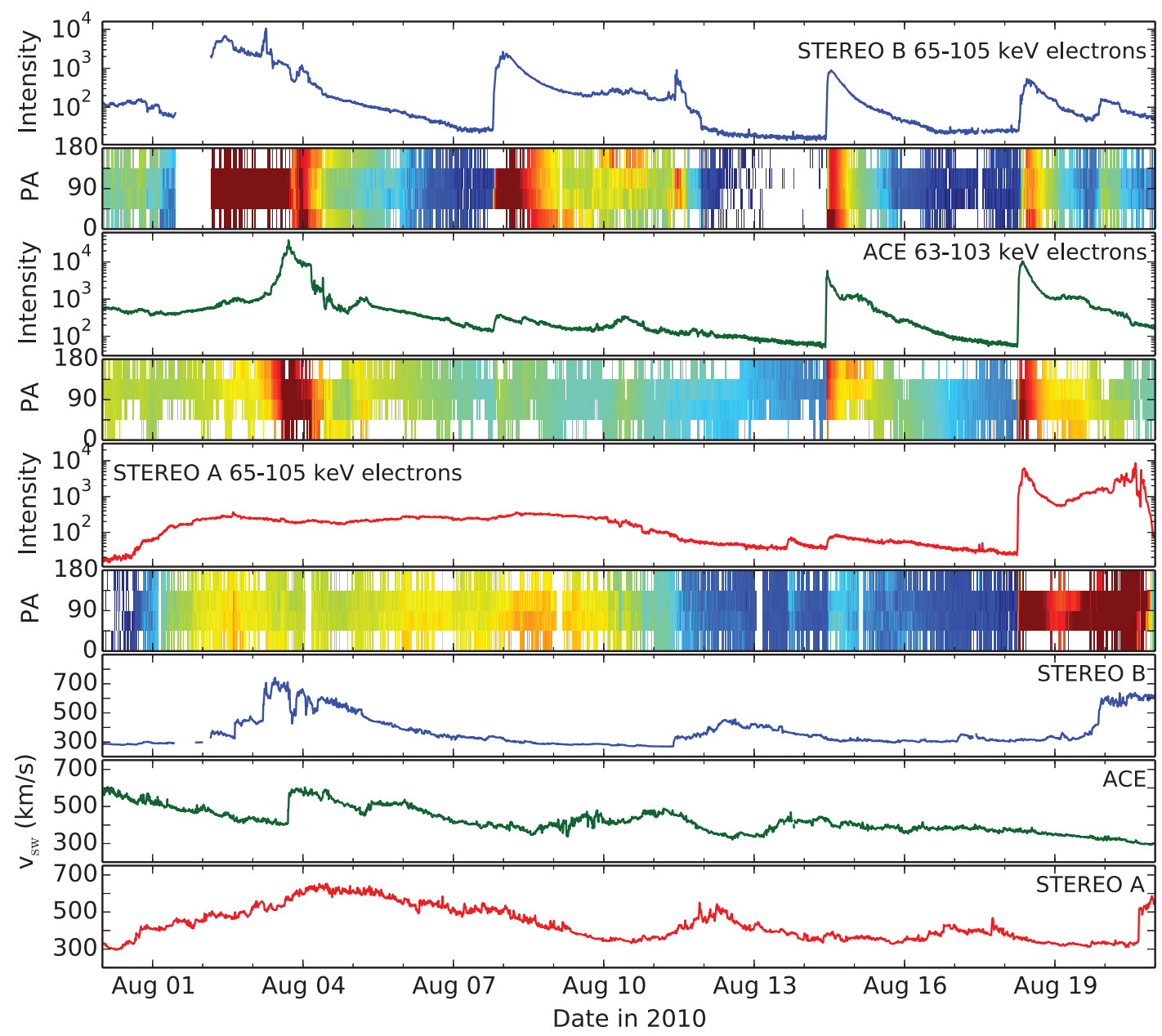

Figure 1: In-situ measurements of $65-105 \mathrm{keV}$ electron intensities and solar wind speeds made by by STEREO-B, ACE and STEREO-A during the time period 30 July to 20 August 2010.

magnetic field line connected with the observer, and subsequently propagate in the solar wind. The latter can usually be described as adiabatic motion along the average interplanetary magnetic field, pitch angle scattering off the fluctuations superimposed on the field, diffusion perpendicular to the average magnetic field due to scattering of the particles' gyrocenter from one field line to another, and due to particles propagating along meandering field lines. The corresponding Fokker-Planck equation which describes the above effects is solved using a time-forward stochastic process with an implicit scheme $[8,9]$. The remaining two stochastic differential equations for the propagation of electrons in a static Parker field under neglecting solar wind effects are then given by

$$
\begin{gathered}
d \boldsymbol{r}(t)=\mu v d t \boldsymbol{b}+\sqrt{2 D_{\perp}} d W_{\perp}(t)+\nabla D_{\perp} d t \\
d \mu(t)=\sqrt{2 D_{\mu \mu}} d W_{\mu}(t)+\left[\frac{v}{2 L}\left(1-\mu^{2}\right)+\frac{\partial D_{\mu \mu}}{\partial \mu}\right] d t
\end{gathered}
$$

where $W_{\mu}(t)$ and $W_{\perp}(t)$ denote one-and two-dimensional Wiener processes, respectively, $\boldsymbol{b}=$ $\boldsymbol{B} / \boldsymbol{B}$ a unit vector in the direction of the average magnetic field $\boldsymbol{B}$. The focusing of particles due to the divergence of the magnetic field is described by focusing length $L(\boldsymbol{r})=-(\boldsymbol{b} \cdot \nabla \ln B(\boldsymbol{r}))^{-1}$. 

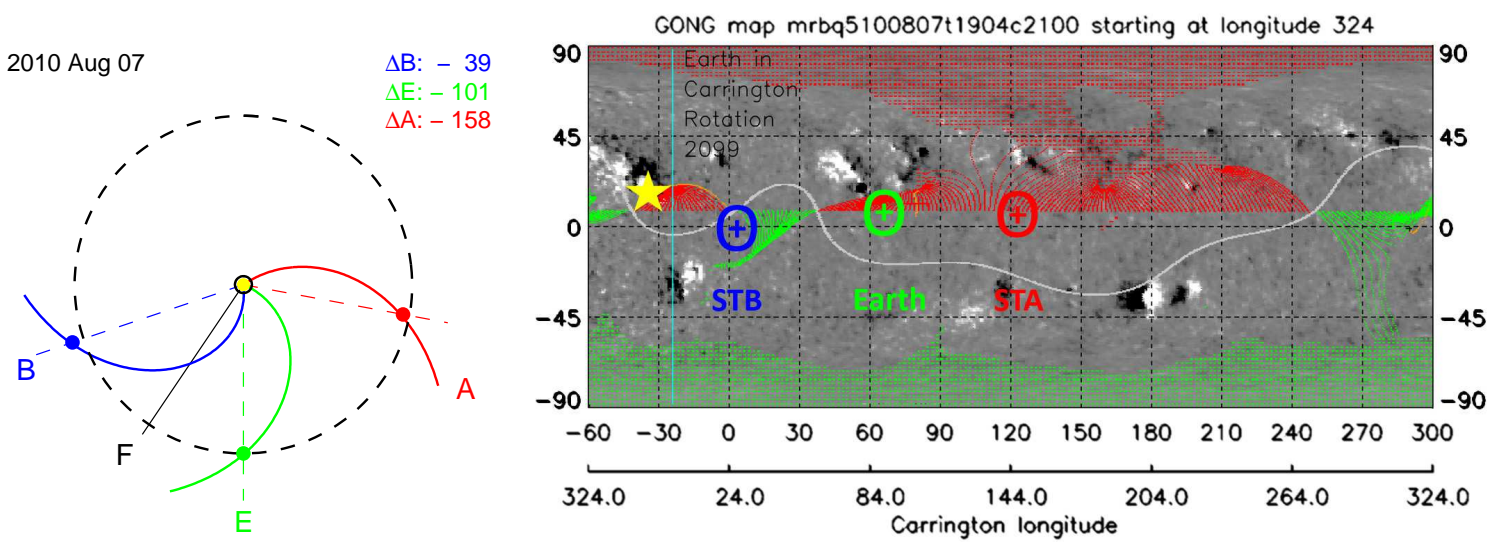

Figure 2: Left panel: Longitudinal positions of the Earth (ACE), STEREO-A and STEREO-B on 2010 August 7. The flare longitude is marked by the solid black line. The longitudinal separations between the spacecraft magnetic foot points and the equatorial footpoints of the magnetic field line from the active region are listed in the upper right corner. Right panel: Synoptic Ecliptic Plane Field Plot overlying a magnetogram map by the Global Oscillation Network Group (GONG) for Carrington rotation 2099. Also marked are the estimated positions of the magnetic foot points of the three spacecraft and the position of the flare.

From the pitch angle diffusion coefficient $D_{\mu \mu}$ a parallel mean free path

$$
\lambda_{\|}(\boldsymbol{r})=\frac{3 v}{8} \int_{-1}^{+1} d \mu \frac{\left(1-\mu^{2}\right)^{2}}{D_{\mu \mu}(\boldsymbol{r}, \mu)}
$$

can be defined which relates the pitch angle scattering rate to the spatial diffusion parallel to the ambient magnetic field. A pitch-angle dependent perpendicular mean free path $\Lambda_{\perp}(\mu)$, and a related perpendicular diffusion coefficient $D_{\perp}(\mu)$ can be formulated from the diagonal elements of the perpendicular diffusion tensor $\boldsymbol{D}_{\perp}(\boldsymbol{r}, p, \mu)$, according to [9]

$$
\Lambda_{\perp}(\mu)=3 / v \cdot D_{\perp}=\alpha \cdot \lambda_{\|}(r) \cdot(r / 1 \mathrm{AU})^{2} \cdot \cos \psi(\mathrm{r}) \cdot \sqrt{1-\mu^{2}} \equiv \alpha \cdot \lambda_{\|} \cdot \Sigma(\mathrm{r}, \psi, \mu)
$$

where $\psi(r)$ is the angle between the magnetic field and the radial direction, and $\alpha$ is a parameter to model the relative contributions of parallel and perpendicular diffusion. The spatial perpendicular diffusion coefficient $K_{\perp}$ and mean free path $\lambda_{\perp}$ which are used in diffusion-convection transport models for isotropic distribution functions, and describe the combined transport effects due to magnetic field line and gyro-center diffusion, can be defined by taking the pitch-angle average of the above equation. Following the lines of our analysis of the transport of electrons in the 2010 February 7 solar particle event [9] we assume that the electrons first undergo lateral transport away from the flare site in complex magnetic fields in the solar corona. When they reach an open magnetic field line they start a three-dimensional transport process as described above. In extension of our earlier analysis of the 2010 February 7 event [9], we here allow a variation of the transport parameters also with heliolongitude.

\section{The 2010 August 7 solar particle event}

The particle event on 2010 August 7 was associated with an M1.0/2F X-ray/optical flare at N15 E37 which started around 18:00 UT. The sketch in the left panel of Figure 2 shows the lon- 

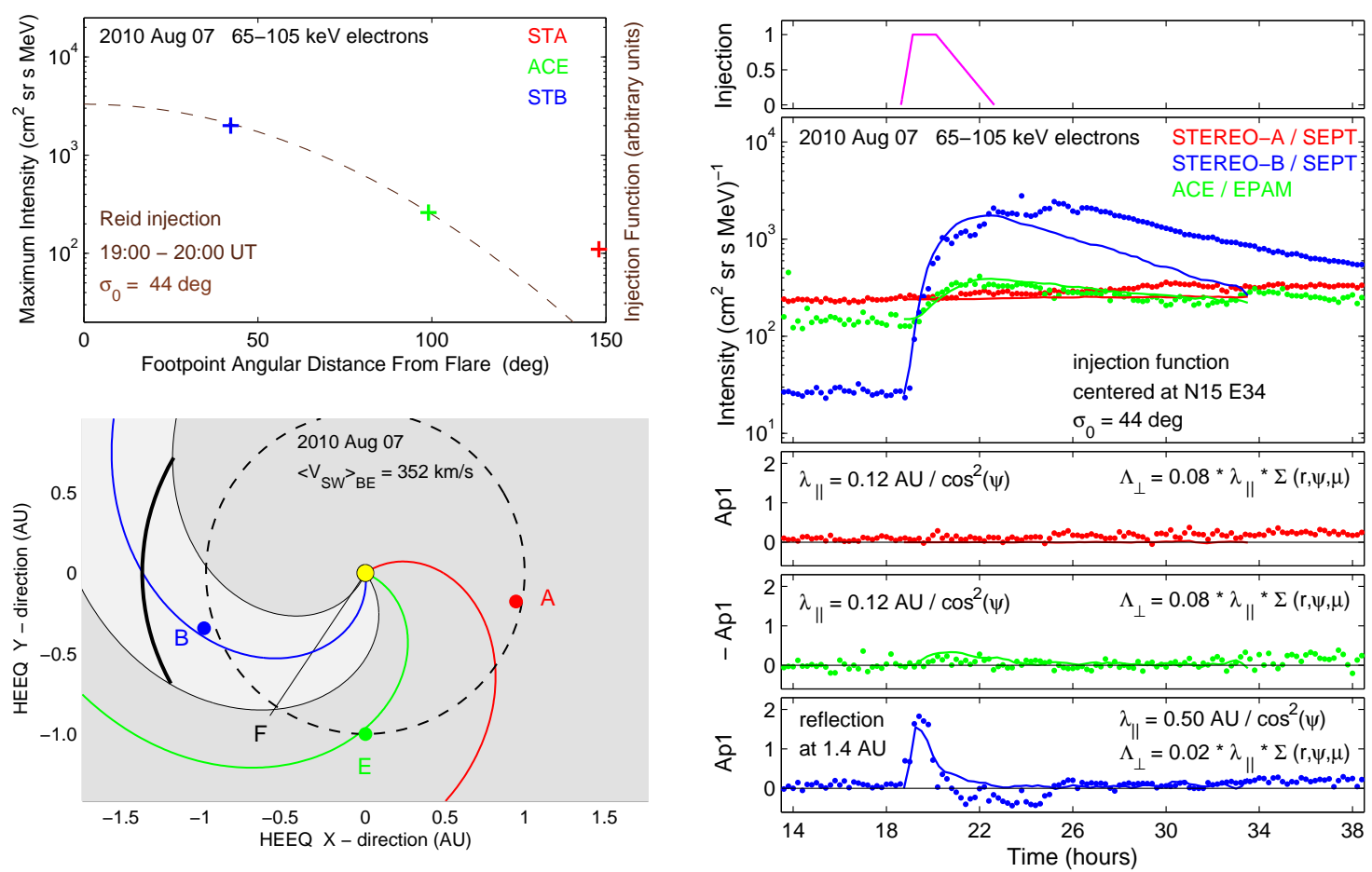

Figure 3: Upper left panel: Angular dependence of electron injection from the solar source surface. Lower left panel: Schematics of longitudinal variation of transport parameters assumed for the three-dimensional modeling of electron transport in the 2010 August 7 solar particle event. Right panel: Comparison of simulation results (solid lines) with electron intensity and anisotropy observations from STEREO-A/B and $A C E$ (dots).

gitudinal positions of the flare and of the three spacecraft on 2010 August 7. STEREO-A and STEREO-B were separated from the Earth-Sun line at 79 degrees and 72 degrees, respectively. Also shown are the magnetic field lines connecting the solar wind source surface and the spacecraft which were obtained assuming a Parker magnetic field. The longitudes $\Phi$ of the magnetic foot points were determined according to $\Phi=\Phi_{0}+\Omega \cdot r / V_{S W}$, where $r$ and $\Phi_{0}$ denote the radial distances and projected longitudes of the spacecraft, respectively, $\Omega$ is the solar rotation frequency, assumed to correspond to a sidereal rotation period of 25.5 days, and $V_{S W}$ is the solar wind speed taken at the time of the event onset at the respective spacecraft. The right panel of Figure 2 shows a Potential Field Source Surface (PFSS) Carrington map overlying a magnetogram map of Carrington rotation 2099, provided by the Global Oscillation Network Group (GONG, http://gong.nso.edu/). The white wavy line represents the neutral sheet, separating the negative polarity sector (marked in red) from the positive polarity sector (marked in green). The flare position is marked by the yellow star, the positions of the estimated positions of the magnetic foot points of the three spacecraft on the solar wind source surface by crosses. We note that due to variations of the solar wind velocity in time and space between the Sun and 1 AU the above positions carry an error of $\sim 10$ degrees (indicated by the circles), and that due to the strong magnetic field in the active region, open field lines can spread to a larger range of longitude and latitude on the source surface.

As one might expect from the relative positions of the flare and the foot points of the field lines connecting to the spacecraft, the maximum electron intensities are highest at STEREO-B, lowest at STEREO-A and in between at ACE. The electron event on STEREO-B (cf., Fig. 3, right) is 

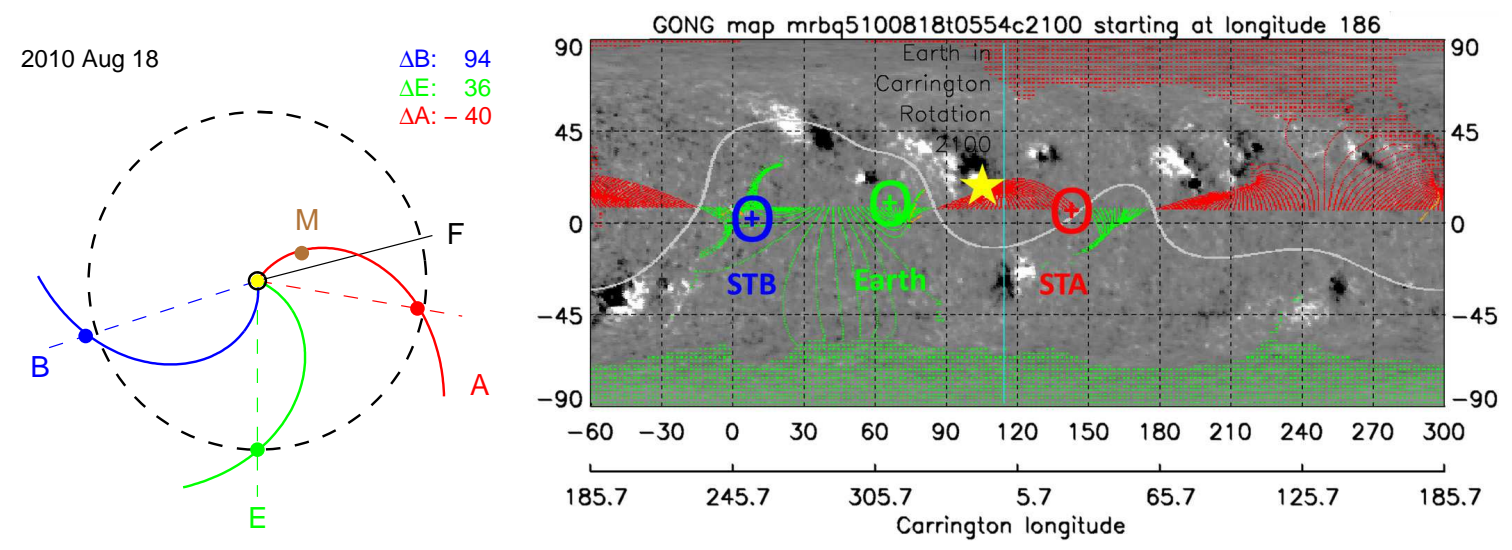

Figure 4: Left: Longitudinal positions of the Earth (ACE), STEREO-A and STEREO-B on 2010 August 18. Right: Synoptic Ecliptic Plane Field Plot overlying a magnetogram map by the Global Oscillation Network Group (GONG) for Carrington rotation 2099, and the estimated positions of the magnetic foot points of the three spacecraft and the position of the flare. Notations as in Fig. 2.

characterized by a fast rise of the intensity and anisotropy profiles. The maximum anisotropy of $\sim 1.8$ indicates a large parallel mean free path. The steep drop in the anisotropy after $\sim$ one hour, followed by a period of slightly negative values, is not consistent with an unobstructed discharge of the electrons from the inner heliosphere but rather suggests a reflection of the particles at an obstacle not too far beyond $1 \mathrm{AU}$. A candidate for such an obstacle could be a CME with an associated shock which could have been released in an event on August 1 (cf., Fig. 1). The fluxes observed on ACE exhibit a gradual rise of the intensity and only a small anisotropy, indicating a small value of $\lambda_{\|}$in the region surrounding ACE. The electron measured on STEREO-A start to slightly exceed the background from a previous event around 03:00 UT on August 8, accompanied by a small rise in the anisotropy. These observations suggest that here none of the accelerated electrons had reached the connecting field line, and that the detected electrons had possibly reached the spacecraft by diffusion perpendicular to the magnetic field in the inner heliosphere. Based on the above, we assume an injection energy spectrum with a power law exponent of 3 , and an injection function $Q(r=0.05 \mathrm{AU}, \sigma, \mathrm{E})=\mathrm{Q}_{0} \mathrm{E}^{-3} \exp \left\{-\sigma^{2} /\left(2 \sigma_{0}^{2}\right)\right\}$ where $\sigma$ is the total angular distance from the acceleration site (assumed to be the location of the flare), and $\sigma_{0}$ characterizes the spread of the electrons on the injection surface close to the Sun. Because our simulations at the current stage can handle only an interplanetary magnetic field structure corresponding to one value of the solar wind speed (equivalent to a rotational symmetry in the equatorial plane) we took for $V_{s w}$ the average of the values observed on STEREO-B and ACE. Simulations for various combinations of the modeling parameters $\lambda_{\|}, \alpha$, and $\sigma_{0}$ were performed. A good overall agreement with the electron observations was found for an injection with $\sigma_{0}=44^{\circ}$ (Fig. 3, upper left) and a time dependence shown in the upper panel of Figure 3, right side. For the parallel and perpendicular mean free paths everywhere except in the magnetic field sector surrounding STEREO-B (see Fig. 3., lower left) values of $\lambda_{\|}=0.12 / \cos ^{2} \psi(\mathrm{r}) \mathrm{AU}$ (equivalent to a radially constant $\lambda_{r}=0.12 \mathrm{AU}$ ) and of $\alpha=0.08$ were assumed. For the sector surrounding STEREO-B we determined $\lambda_{\|}=0.5 / \cos ^{2} \psi(\mathrm{r})$ AU (equivalent to $\lambda_{r}=0.5 \mathrm{AU}$ ) and $\alpha=0.02$. For this sector also a boundary at $r=1.4 \mathrm{AU}$ with a reflection probability of $90 \%$ had to be assumed. Panels 2-5 of Figure 3 (right) show that the results of the simulation reproduce the observed intensity and anisotropy profiles reasonably well. 

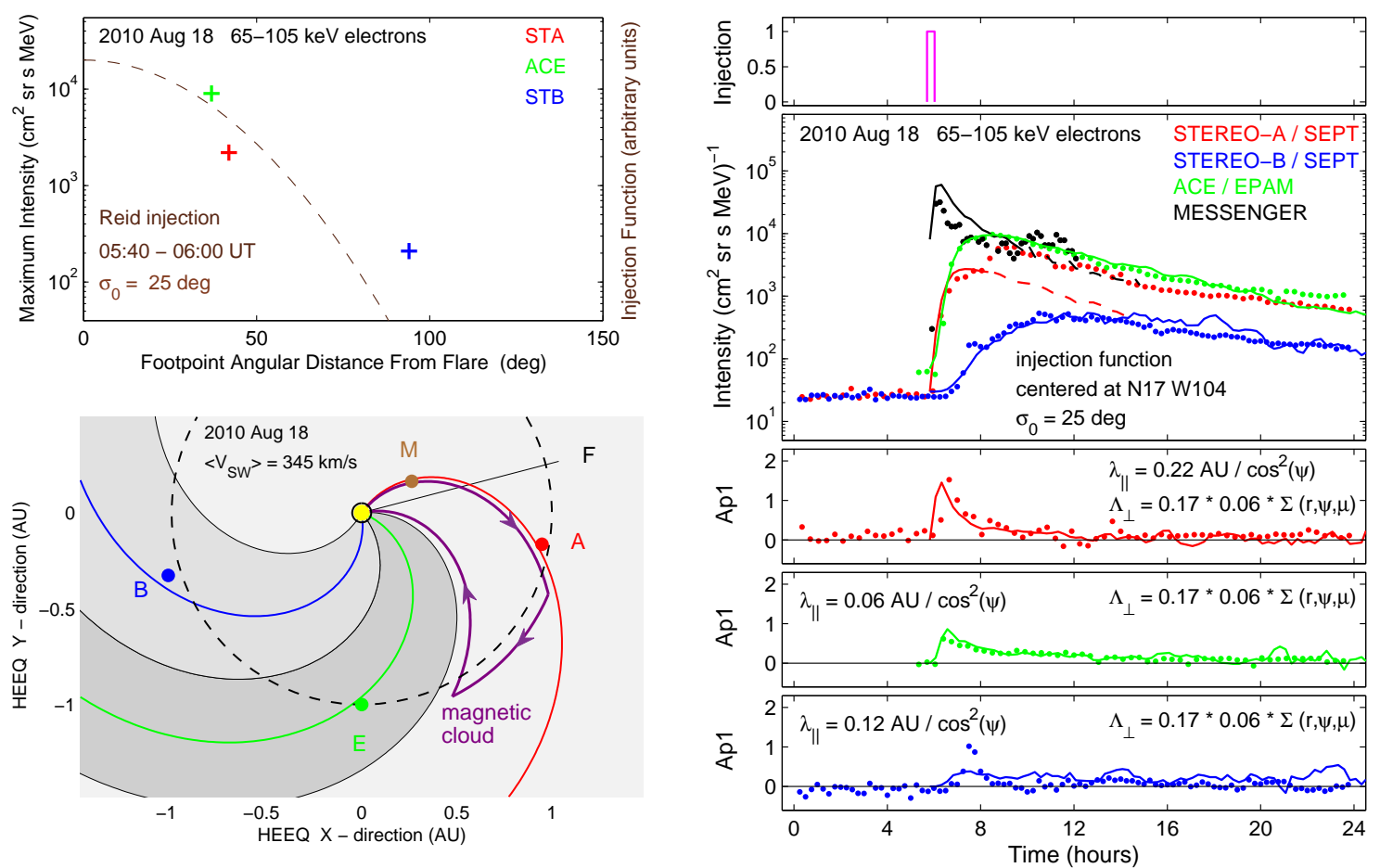

Figure 5: Upper left: Angular dependence of electron injection from the solar source surface. Lower left: Longitudinal variation of the radial mean free path assumed for the three-dimensional modeling of electron transport in the 2010 August 18 solar particle event. Right, top to bottom: injection function, comparison of simulation results (solid lines) with electron intensity and anisotropy observations on $S T E R E O-A / B$ and ACE (dots).

\section{The 2010 August 18 solar particle event}

The particle event on 2010 August 18 was associated with a C4.5 X-ray flare occurring slightly behind the west limb $\left(\sim 20^{\circ}\right.$, as viewed from Earth), starting around 04:45 UT. In a similar way as before, Figure 4 shows the longitudinal positions of the flare and of the three spacecraft, as well as a GONG map of Carrington rotation 2099 on which the locations of the flare and the estimated positions of the magnetic foot points of the three spacecraft are marked. A previous study of the August 18 particle event [10] reported that the structure of the interplanetary magnetic field at that time deviated strongly from regular conditions. In particular, it was found from plasma properties and the observation of large bi-directional anisotropies that STEREO-A had entered a magnetic cloud with about one and a half hours after the flare onset in which it stayed until 05:00 on August 19. To allow for a variation of transport conditions as a function of longitude we define sectors in the ecliptic plane around STEREO-B, ACE, and the remaining area including STEREO-A and the magnetic cloud (see sketch in Fig. 5 bottom left). Figure 5 (right) shows that the intensities on STEREO-A and ACE start to rise simultaneously. On STEREO-A they reach a first maximum which is a factor of $\sim 3$ below the maximum on ACE, and start to rise again after entering the magnetic cloud. As our simulation is not able to handle short-time reconfigurations of the magnetic field, we decided to model the electron observations on STEREO-A only in the early phase when the spacecraft is not yet in the magnetic cloud. Similar as for the August 7 event we tried out various parame- 
ter combinations, and found a best overall agreement for the following values: a short injection (Fig. 5, upper right) with $\sigma_{0}=25^{\circ}$ (Fig. 5, upper left), and parallel mean free path in the magnetic field sectors surrounding STEREO-A, ACE, STEREO-B of $\lambda_{\|}=(0.22 / 0.06 / 0.12) / \cos ^{2} \psi(\mathrm{r})$ AU, respectively. For the perpendicular mean free path we assumed a longitudinally independent value of $\Lambda_{\perp}=0.17 \cdot 0.06 \cdot \Sigma(r, \psi, \mu)$. The results of the simulation reproduce the observed intensity and anisotropy profiles (Fig. 5 right, panels 2-5) well, with the exception of the anisotropy observed on STEREO-B where we have not been able to model the high values between 07:30 and 08:00 UT for any reasonable combination of transport parameters. A possible explanation could be that the spacecraft was temporarily in a magnetic flux tube with a larger value of $\lambda_{\|}$and a better connection to the flare. A comparison of MESSENGER observations [11] of electrons in a similar energy range with the prediction from the simulation for the STEREO-A sector at $0.3 \mathrm{AU}$ (black dots and line) shows a good agreement in the shapes of the intensity profiles, whereas there is a difference of a factor of $\sim 2$ in the absolute values. The latter could be due to the fact that the exact pitch angles of the MESSENGER electrons are not known and that the electron transport here could also have been affected by the magnetic cloud, as indicated by the intensity increase observed after $\sim$ 09:00 UT.

\section{Conclusions}

In this work we have presented transport modelings of two solar electron events observed by STEREO-A/B and ACE in August 2010 which were produced by a pair of active regions as they rotated around the Sun. We have demonstrated that our simulation method can handle advanced features of interplanetary propagation recently found in wide spread particle events ([12]), e.g., a longitudinal variation of the transport parameters and the effects of disturbances in the solar wind during times of high solar activity, and that it allows to disentangle various effects involved in the particle transport, such as diffusion parallel and perpendicular to the interplanetary magnetic field, and in the magnetic field structures in the solar corona.

The STEREO/SEPT projects are supported under Grant 50 OC 1302 by the German Bundesministerium für Wirtschaft through the Deutsches Zentrum für Luft- und Raumfahrt (DLR). We acknowledge the ACE and STEREO teams for providing the data used in this work.

\section{References}

[1] R. Müller-Mellin, S. Böttcher, J. Falenski, et al., Space Sci. Rev. 136 (2008) 363

[2] J. G. Luhmann, D. W. Curtis, P. Schroeder, et al., Space Sci. Rev. 136 (2008) 117

[3] R. E. Gold, et al., Space Sci. Rev. 86 (1998) 541

[4] M. H. Acuna, D. Curtis, J. L. Scheifele, et al., Space Sci. Rev. 136 (2008) 203

[5] C. W. Smith, J. L.'Heureux, N. F. Ness, et al., Space Sci. Rev. 86 (1998) 86, 613

[6] A. B. Galvin, L. M. Kistler, M. A. Popecki, et al., Space Sci. Rev. 136 (2008) 437

[7] D. J. McComas, S. J. Bame, P. Barker, et al., Space Sci. Rev. 86 (1998) 563

[8] W. Dröge, Y. Y. Kartavykh, B. Klecker, G. A. Kovaltsov, ApJ 709 (2010) 912

[9] W. Dröge, Y. Y. Kartavykh, N. Dresing, B. Heber, A. Klassen, JGRA 119(8) (2014) 6074

[10] R. A. Leske, C. M. S. Cohen, R. A. Mewaldt, et al., Solar Phys 281 (2012) 301

[11] D. Lario, et al., http://www.srl.caltech.edu/ACE/ACENews/ACENews139.html (2011)

[12] N. Dresing, et al., A\&A 567 (2014) A27 